\title{
AN ANALYTICAL APPROACH TO THE SPIN DISTRIBUTION OF DARK HALOS
}

\author{
TZIHONG CHIUEH \\ Department of Physics, National Taiwan University, 1 Roosevelt Road, Section 4, Taipei, Taiwan; \\ and Institute of Astronomy and Astrophysics, Academia Sinica, P.O. Box 23-141, Taipei 106, Taiwan; \\ chiuehth@phys.ntu.edu.tw \\ JOUNGHUN LEE \\ Institute of Astronomy and Astrophysics, Academica Sinica, P.O. Box 23-141, Taipei 106, Taiwan; \\ and Department of Physics, University of Tokyo, 7-3-1 Hongo, Bunkyo-ku, Tokyo 113-0033, Japan; \\ lee@utap.phys.s.u-tokyo.ac.jp \\ AND \\ LIHWAI LIN \\ Department of Physics, National Taiwan University, 1 Roosevelt Road, Section 4, Taipei, Taiwan; \\ d90222005@ms90.ntu.edu.tw \\ Received 2002 May 2; accepted 2002 August 19
}

\begin{abstract}
We derive the distribution of the dimensionless specific angular momentum of dark matter halos $P(j)$ in the frameworks of tidal-torque theory as well as the nonspherical collapse model and explain the characteristic shape of $P(j)$ commonly observed in $N$-body simulations. A scalar quantity (shear scalar) $r$ is introduced for measuring the effective strength of the tidal-torque force acting on the halo from the surrounding matter. It is found that the ubiquitous and broad shape of $P(j)$ can be mostly attributed to the unique property of the shear scalar $r$ and also that $P(j)$ is in fact insensitive to the underlying collapse dynamics. Our result demonstrates that although the shape of $P(j)$ is ubiquitous and close to lognormal, the distribution is not exactly lognormal, but decreases exponentially at the high angular momentum end and drops as a power law with an index of 2 at the low angular momentum end.
\end{abstract}

Subject headings: cosmology: theory — large-scale structure of universe

\section{INTRODUCTION}

Galaxy spin is believed to be coupled strongly to the host dark matter halo. The halo rotation is, however, inaccessible to measurements, except by some indirect means (Chiueh \& Tseng 2000). Hence, the only viable and powerful way for investigation of the origin of galaxy angular momentum has been through $N$-body simulations. Highresolution $N$-body simulations commonly reveal that dark matter halos have an intrinsic angular momentum distribution with a broad and ubiquitous shape, independent of other halo properties such as mass, formation epoch, and so on (Barnes \& Efstathiou 1987; Heavens \& Peacock 1988; Lemson \& Kauffmann 1999; Bullock et al. 2001; Chen \& Jing 2002). This characteristic distribution of halo angular momentum is often approximated by a lognormal distribution with a ubiquitous mean and variance in the log. Although it has been shown that this characteristic distribution can be produced by the current standard model (Maller, Dekel, \& Somerville 2002), the following important questions have not yet been answered. What is the physical origin of the characteristic distribution? It is known that the lognormal distribution often results from highly nonlinear random processes. What are the mechanisms underlying this lognormal behavior?

The standard model for the origin and evolution of halo angular momenta is the tidal-torque theory (Doroshkevich 1970; White 1984). In this model, the angular momentum of a protohalo, $\boldsymbol{L}$, is generated by misalignment among the principal axes of the inertia tensor and those of the local shear tensor and grows with time in the Lagrangian coordinate $\boldsymbol{q}$ as $L_{i}(t)=-a^{2}(t) \dot{D}(t) \epsilon_{i j k} \mathrm{~T}_{j l} \mathrm{I}_{l k}$. Here, $a(t)$ is the uni- versal scale factor, $D(t)$ is the growing factor of density perturbations, and $\mathrm{I}_{l k} \equiv \int \rho_{0} q_{l} q_{k} d^{3} \boldsymbol{q}$, with $\rho_{0}$ being the mean density of the universe at the epoch of $a(t)=1$, where $\mathrm{I}_{l k}$ is the inertia tensor of the protohalo, characterizing the halo geometrical shape. In addition, $\mathrm{T}_{j l} \equiv \partial^{2} \phi / \partial q_{j} \partial q_{l}$, where $\phi$ is the gravitational potential that satisfies the Poisson equation $\nabla_{q}^{2} \phi(\boldsymbol{q})=4 \pi G \delta(\boldsymbol{q}, t) D^{-1}(t)$, with $\delta$ being the density contrast; $\mathrm{T}_{j l}$ is the gravitational shear tensor, quantifying the tidal torque from the surrounding matter.

Tidal-torque theory was conceived as a linear theory describing the linear evolution of angular momentum and should break down in the nonlinear regime. To one's surprise, however, it has been demonstrated by $N$-body simulations that tidal-torque theory works well even in the nonlinear regime (Frenk et al. 1988; Heavens \& Peacock 1988; Catelan \& Theuns 1996; Sugerman, Summers, \& Kamionkowski 2000; Maller et al. 2002; Porciani, Dekel, \& Hoffman 2002a, 2002b). This nontrivial result indicates that this seemingly linear theory contains relevant elements of nonlinear physics. Indeed, to identify those collapsed particles in the Lagrangian space $\boldsymbol{q}$ that determine the inertia tensor $\mathrm{I}_{i j}$, knowledge of nonlinear evolution is required. Moreover, in the context of angular momentum in a collapsed halo, tidal-torque theory must be extended to combine with collapse dynamics. In the Press-Schechter model, collapse dynamics is represented by the spherical collapse condition, and for a more realistic nonspherical collapse, the corresponding collapse conditions have also been identified (Sheth, Mo, \& Tormen 2001; Sheth \& Tormen 2002; Chiueh \& Lee 2001; Lin, Chiueh, \& Lee 2002). Hence, the tidal-torque theory in its extended form, as given below, does contain significant nonlinear physics. We thus adopt 
the tidal-torque theory as well as the nonspherical collapse model as the basic framework for the investigation of the halo angular momentum distribution.

Section 2 provides a new mathematical framework to express the halo angular momentum in terms of five relevant random variables. Section 3 presents our predicted halo angular momentum distribution. Discussions and conclusions are given in $\S 4$.

\section{MATHEMATICAL FRAMEWORK}

For the distribution of halo angular momentum, it is more convenient to use the manifestly mass-independent specific angular momentum $j$, rather than the angular momentum $L$ itself, which scales with the halo mass as $L \sim I \sim \int q^{2} d^{3} \boldsymbol{q} \sim R^{5} \sim M^{5 / 3}$, with $R$ and $M$ being the typical precollapse size and mass of a protohalo, respectively. We thus define the specific angular momentum as $j_{i} \equiv \epsilon_{i j k} \mathrm{~T}_{j l} \overline{\mathbf{I}}_{l k}$, with $\overline{\mathbf{I}} \equiv \mathbf{I} M^{-5 / 3}$. Since the $\log (j)$ distribution is to be evaluated at the same epoch, it has the same shape as the $\log (L)$ distribution, apart from a constant horizontal shift.

Rotating the coordinates into the local shear principalaxis frame, we find that the magnitude of the specific angular momentum is given as

$j=\left[\overline{\mathbf{I}}_{23}^{2}\left(\lambda_{2}-\lambda_{3}\right)^{2}+\overline{\mathbf{I}}_{31}^{2}\left(\lambda_{1}-\lambda_{3}\right)^{2}+\overline{\mathbf{I}}_{12}^{2}\left(\lambda_{1}-\lambda_{2}\right)^{2}\right]^{1 / 2}$,

where $\lambda_{1}, \lambda_{2}$, and $\lambda_{3}$ are the three eigenvalues of the local shear tensor and $\overline{\mathbf{I}}_{23}, \overline{\mathbf{I}}_{31}$, and $\overline{\mathbf{I}}_{12}$ are the three off-diagonal components of the dimensionless inertia tensor $\overline{\mathbf{I}}$. We stress that in contrast to the conventional notion in which the eigenvalues are ordered as $\lambda_{1} \geq \lambda_{2} \geq \lambda_{3}$, we do not order the eigenvalues in equation (1), and the subscripts of the $\lambda$ 's are only meant to denote different directions of unordered shear principal axes. As these $\lambda$ 's are unordered, the three random eigenvalues are indistinguishable and obey the same statistics. The same argument also applies to the three off-diagonal components of $\mathbf{I}_{i j}$.

One notes that when the shear tensor and inertia tensor are perfectly aligned, the dynamics involve only a potential flow, much like the gravitational collapse of a single ellipsoid. However, it is only when the shear tensor and inertia tensor are misaligned that rotational motion can be generated and angular momentum transferred in between the surrounding matter and collapsing halos. Interestingly, recent $N$-body simulations have shown that the shear and inertia tensors are strongly, but not perfectly, aligned (Lee \& Pen 2000; Porciani et al. 2002a, 2002b). That is, in the shear principal axes, the magnitudes of the inertia off-diagonal components are small relative to the diagonal parts. This numerical evidence for the small off-diagonal components of the inertia tensor suggests to us that the misalignment is statistical fluctuation, and $\overline{\mathbf{I}}_{23}, \overline{\mathbf{I}}_{31}$, and $\overline{\mathbf{I}}_{12}$ can be regarded as independent random variables. Since the three eigenvalues of the shear tensor are also random variables, equation (1) describes how the positive random variable $j$ depends on the random variables of off-diagonal $\overline{\mathbf{I}}$ and $\lambda_{i}$.

To derive the distribution of $j$, we first separate these variables as follows: We view the generation of the halo angular momentum as a $3 \times 3$ diagonal tidal tensor $\mathrm{R}_{i j} \equiv \operatorname{Diag}\left[r_{1}, r_{2}, r_{3}\right]$ acting on an inertia-moment vector
$\tilde{\boldsymbol{I}}$, i.e., $j_{i}=\sqrt{3} \mathrm{R}_{i j} \tilde{I}_{j}$, in the frame of the local shear principal axis, where the components of $\mathrm{R}_{i j}$ and $\tilde{\boldsymbol{I}}$ are given as $r_{1}=$ $\left(\lambda_{2}-\lambda_{3}\right) / \sqrt{3}, r_{2}=\left(\lambda_{3}-\lambda_{1}\right) / \sqrt{3}$, and $r_{3}=\left(\lambda_{1}-\lambda_{2}\right) / \sqrt{3}$, and $\tilde{I}_{1} \equiv \tilde{I}_{23}, \tilde{I}_{2} \equiv \overline{\mathrm{I}}_{31}$, and $\tilde{I}_{3} \equiv \overline{\mathbf{I}}_{12}$. With the three components of $\tilde{\boldsymbol{I}}$ being independent random variables, we can further express them in spherical coordinates as $\tilde{I}_{\tilde{L}}=$ $\tilde{I} \sin \theta \cos \phi, \tilde{I}_{2}=\tilde{I} \cos \theta$, and $\tilde{I}_{3}=\tilde{I} \sin \theta \sin \phi$, with $\tilde{I} \equiv$ $\left(\tilde{I}_{1}^{2}+\tilde{I}_{2}^{2}+\tilde{I}_{3}^{2}\right)^{1 / 2}, \theta \equiv \cos ^{-1}\left(\tilde{I}_{2} / \tilde{I}\right)$, and $\phi \equiv \tan ^{-1}\left(\tilde{I}_{3} / \tilde{I}_{1}\right)$. On the other hand, the diagonal components of $\mathrm{R}_{i j}$ are not all free but are constrained by the condition $r_{1}+r_{2}+r_{3}=0$. One can conveniently also regard the diagonal components $\left(r_{1}, r_{2}, r_{3}\right)$ as a vector $\boldsymbol{r}$; this vector lies in a plane perpendicular to the $(1,1,1)$ symmetric axis in $\lambda$ space, or $\boldsymbol{r}=\lambda(1,1,1) / \sqrt{3}$. Therefore, $\boldsymbol{r}$ depends only on one polar angle, for instance, $\psi$.

To find $\boldsymbol{r}$ expressed in polar coordinates, we perform a coordinate transformation from $\left(\lambda_{1}, \lambda_{2}, \lambda_{3}\right)$ to $(x, y, \delta)$ with $x \equiv\left(\lambda_{1}-2 \lambda_{2}+\lambda_{3}\right) / \sqrt{6}, \quad y \equiv\left(\lambda_{1}-\lambda_{3}\right) / \sqrt{2}, \quad \delta \equiv \lambda_{1}+\lambda_{2}$ $+\lambda_{3}$. Under this transformation, the transformed $\boldsymbol{r}^{\prime}$ has only two components: $\boldsymbol{r}^{\prime}=(x, y, 0)$, with $x=r \cos \psi$, $y=r \sin \psi$. Now, rotating the axes back into the shear principal axes, we have a polar angle expression for $\boldsymbol{r}$ in the shear principal-axis frame: $r_{1}=-(1 / 2)^{1 / 2} r \cos \psi+(1 / 6)^{1 / 2}$ $\times r \sin \psi, \quad r_{2}=-(2 / 3)^{1 / 2} r \sin \psi, \quad r_{3}=(1 / 2)^{1 / 2} r \cos \psi$ $+(1 / 6)^{1 / 2} r \sin \psi$.

Using these angular variables for $\tilde{\boldsymbol{I}}$ and $\boldsymbol{r}$, we find the following expression for $j$ with separated variables:

$$
j=\tilde{I} r Y(\psi, \theta, \phi),
$$

where

$$
\begin{aligned}
Y(\psi, \theta, \phi) \equiv & {\left[\operatorname { s i n } ^ { 2 } \theta \left(\frac{1}{2} \cos ^{2} \psi+\frac{1}{6} \sin ^{2} \psi\right.\right.} \\
& \left.-\frac{1}{2 \sqrt{3}} \cos 2 \phi \sin 2 \psi\right) \\
& \left.+\frac{2}{3} \cos ^{2} \theta \sin ^{2} \psi\right]^{1 / 2} .
\end{aligned}
$$

To derive the distribution of $j$ using equation (2), we further assume that the off-diagonal components of the inertia tensor are Gaussian variables. In fact, determination of the inertia tensor distribution is quite difficult without knowing the exact Lagrangian boundary of the collapsed halos. It may seem that in Gaussian peak formalism, in which halos are condensed out from the local maxima of the initial Gaussian density field, the inertia tensors are related to the second derivative of the Gaussian density field, in which case the assumption of the Gaussian inertia tensor components is justified (Heavens \& Peacock 1988; Catelan \& Theuns 1996). However, in the present context, it is the offdiagonal components of the inertia tensor in a particular frame, the shear principal-axis frame, that are in question, and hence the above argument for the Gaussianity of $\tilde{I}_{i}$ cannot be justified. Instead, we conceive that halo formation processes involve chaotic dynamics, which makes the halo Lagrangian boundary that determines the inertia tensor rather irregular. Without detailed knowledge of the chaotic dynamics, we simply assume the Gaussianity of $\tilde{I}_{i}$ as an approximation without further justification. This assumption is testable by cosmological $N$-body simulations.

With this assumption, the distribution of $\tilde{I}$ $\left[\equiv\left(\tilde{I}_{1}^{2}+\tilde{I}_{2}^{2}+\widetilde{I}_{3}^{2}\right)^{1 / 2}\right]$ becomes a weighted Gaussian given 
by $P_{\tilde{I}}(\tilde{I}) d \tilde{I}=(2 / \pi)^{1 / 2} \sigma_{\tilde{I}}^{-3} \tilde{I}^{2} \exp \left(-\tilde{I}^{2} / 2 \sigma_{\tilde{I}}^{2}\right) d \tilde{I}$, where $\sigma_{\tilde{I}}$ is the standard deviation of each off-diagonal component. Again, since the shear principal axes are unordered, each off-diagonal component of $I$ in the shear principal-axis frame has been given the same standard deviation $\sigma_{\tilde{I}}$. Due to the isotropic $\tilde{I}_{i}$, the solid angles $\phi$ and $\theta$ are uniformly distributed on a sphere.

\section{DISTRIBUTION OF ANGULAR MOMENTUM}

\section{1. r-Distribution}

Although the shear scalar $r$ was first pointed out by Chiueh \& Lee (2001) to play an important role in nonspherical halo formation and angular momentum generation, Sheth \& Tormen (2002) recognized that $r$ can in fact be conveniently expressed as $\left[(2 / 15) \sum_{i=1}^{5} y_{i}\right]^{1 / 2}$, where $y_{1}, \ldots, y_{5}$ are mutually independent Gaussian variables, having the same standard deviations as that of the density field, $\sigma_{\delta}$. Therefore, the distribution of $r$ is also a weighted Gaussian: $P_{r}(r)=(5 / \pi)^{1 / 2}\left(25 / 12 \sigma_{\delta}^{5}\right) r^{4} \exp \left(-5 r^{2} / 4 \sigma_{\delta}^{2}\right)$. Sheth \& Tormen (2002) commented that the broadness of $P(j)$ may be attributed to the shape of $P_{r}(r)$. However, $P(j)$ is in fact determined by the conditional distribution of $r$ only for those regions where dark halos condense, i.e., $P_{r}(r \mid$ halo $)$, instead of the unconditional $P_{r}(r)$ given above. In the PressSchechter model of halo formation (Press \& Schechter 1974), in which the collapse threshold depends only on $\delta$ and not on $r, P_{r}(r \mid$ halo $)$ indeed equals $P_{r}(r)$. However, the actual halo collapse is generally nonspherical, and it leads to higher collapse thresholds that depend on both $\delta$ and $r$ (Sheth et al. 2001; Chiueh \& Lee 2001). This fact differentiates the actual halo $r$-distribution per mass bin, $P_{r}(r \mid$ halo $)$, from the unconditional $P_{r}(r)$.

To fix $P_{r}(r \mid$ halo), we examine the nonspherical collapse using the collapse condition given by Chiueh \& Lee (2001): $\delta / \delta_{c}=\left(1+r^{4} / \alpha\right)^{\beta}$. Chiueh \& Lee (2001) described the formation of dark halos via nonspherical collapse as a diffusion-like random walk process in the three-dimensional shear principal $\left(\lambda_{1}, \lambda_{2}, \lambda_{3}\right)$ coordinate and determined the nonspherical condition for halo formation by employing the excursion set approach (Sheth \& Tormen 2002). The diffusion in three-dimensional space was solved numerically with the help of Monte Carlo simulations. By adjusting the values of $\alpha$ and $\beta$ for the collapse condition, it reproduces the currently popular Sheth-Tormen mass function (Sheth \& Tormen 1999).

Chiueh \& Lee (2001) originally obtained $\alpha=\beta=0.15$; recently, Lin et al. (2002) refined the values to be $\alpha=0.26$ and $\beta=0.16$. In fact, it was shown by Sheth \& Torman (2002) that the mass function is rather insensitive to the exact collapse condition, and motivated by this, Lin et al. (2002) used the condition mass function to constrain the collapse condition.

The refined values of $\alpha$ and $\beta$ are adopted here. We calculate $P_{r}(r \mid$ halo $)$ numerically using the random walk method described in Lin et al. (2002), which instead of directly computing $\lambda$ 's by diagonalizing the shear tensor, employs the much more efficient $y_{j}$-variable representation mentioned earlier. The efficiency of the $y_{j}$-variable representation allows us to examine the $r$-distribution in detail. Two nontrivial results are found. First, $P_{r}(r \mid$ halo $)$ is independent of mass up to numerical accuracy, and second, $P_{r}(r)$ turns out to still be a good approximation to $P_{r}(r \mid$ halo $)$.

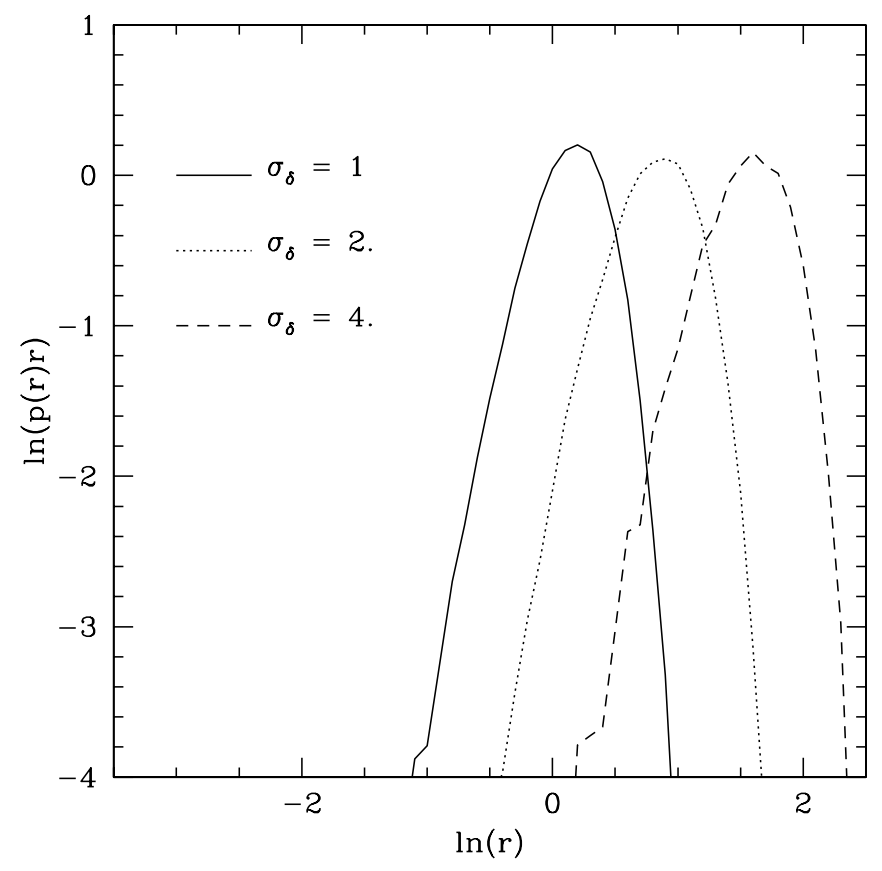

FIG. 1.-Distribution of the halo shear scalar $r$ in the logarithmic scale at three different values of the rms density fluctuations $\sigma_{\delta}$.

In Figure 1 we plot $P_{r}(r \mid$ halo $)$ at three different values of the density rms fluctuation $\sigma_{\delta}$ (equivalently, halo mass) in a logarithmic scale. As shown, the shape of $P_{r}(r \mid$ halo $)$ is selfsimilar to a high degree of approximation, irrespective of the value of $\sigma_{\delta}$. Moreover, the peak positions change linearly in $\ln (r)$, showing that the random number $r$ scales with $\sigma_{\delta}$ linearly. In $\Lambda$-dominated cold dark matter cosmology, with $\Omega_{\Lambda}=0.7$ and $\Omega_{m}=0.3$, this range $\sigma_{\delta}=1-4$ corresponds to a mass range from $1.6 \times 10^{14}$ to $3.5 \times 10^{10} h^{-1}$ $M_{\odot}$. Because of the linear dependence of $r$ on $\sigma_{\delta}$ as well as the self-similar shape of $P_{r}(r \mid$ halo), we choose a particular $\sigma_{\delta}$ for the following comparison. Figure 2 compares the numerical $P_{r}(r \mid$ halo $)$ near the characteristic mass $M$ * $\left(\sigma_{\delta}=1.69\right)$ with the unconditional $P_{r}(r)$ given above. The good agreement between the two suggests that the distribution of $j$ is not sensitive to the detailed dynamics of halo formation. This feature is the origin of the scale-independent shape of the halo angular momentum distribution to be shown below.

\section{2. $j$-Distribution}

Approximate $P_{r}(r \mid$ halo $)$ by the simpler $P_{r}(r)$. By equation (2), the angular distribution $P(j)$ can be derived as

$$
\begin{aligned}
P(j)= & \int d \lambda_{1} d \lambda_{2} d \lambda_{3} \int d \tilde{I} \sin \theta d \theta d \phi \\
& \times P_{\lambda}\left(\lambda_{1}, \lambda_{2}, \lambda_{3}\right) P_{\tilde{I}} \delta_{\mathrm{D}}(j-\tilde{I} r Y(\theta, \phi, \psi)),
\end{aligned}
$$

where

$$
\begin{aligned}
P_{\lambda}\left(\lambda_{1}, \lambda_{2}, \lambda_{3}\right)= & \frac{3375}{8 \sqrt{5} \pi \sigma_{\delta}^{6}} \exp \left(-\frac{\delta^{2}}{2 \sigma_{\delta}^{2}}-\frac{5 r^{2}}{4 \sigma_{\delta}^{2}}\right) \\
& \times\left|\left(\lambda_{1}-\lambda_{2}\right)\left(\lambda_{2}-\lambda_{3}\right)\left(\lambda_{1}-\lambda_{3}\right)\right|,
\end{aligned}
$$

and the Dirac $\delta_{\mathrm{D}}$-function constrains $j$ to other variables through equation (2). Note that the last factor in equation 


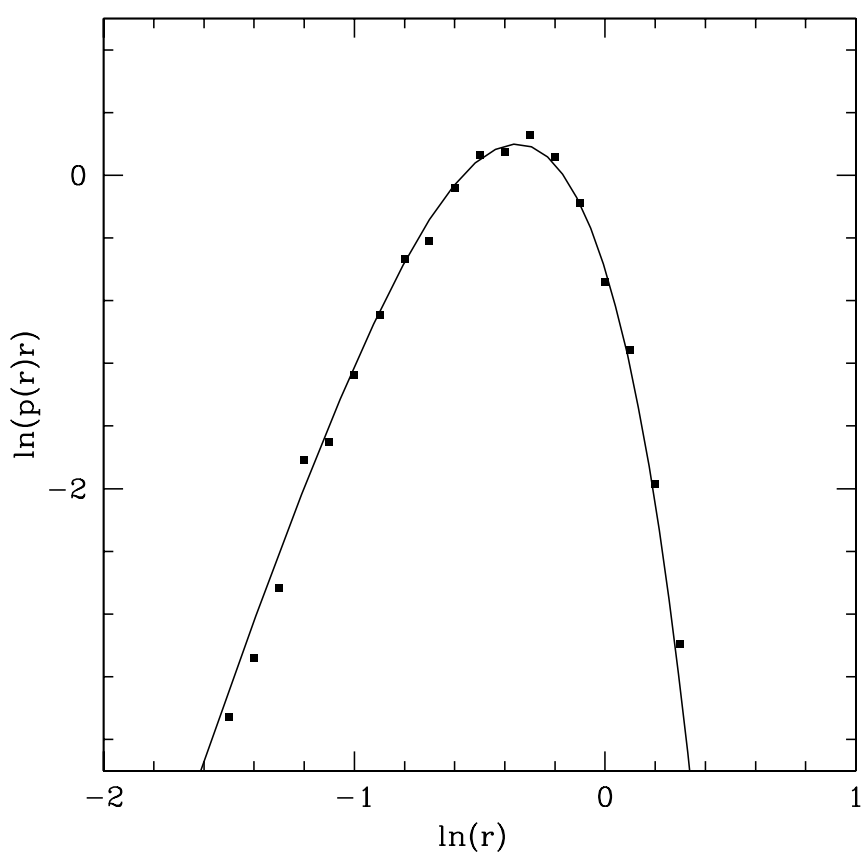

FIg. 2.-Distribution of the halo shear scalar $r$ in the logarithmic scale. The squares show $P_{r}(r \mid$ halo $)$ near $M *$ obtained from a Monte Carlo simulation using the nonspherical collapse condition. The solid curve shows $P_{r}(r)$ given by an analytic formula with the Press-Schechter collapse condition.

(5) is no more than $\left|r_{1} r_{2} r_{3}\right|[\equiv J(r, \psi)]$, arising from the Jacobian of the angular distribution of the shear principal-axis coordinate (Doroshkevich 1970). Thus, $J(r, \psi)=r^{3} J(\psi)$, with $J(\psi) \equiv\left|\left(3 \cos ^{2} \psi-\sin ^{2} \psi\right) \sin \psi\right|$. The angular dependence of $J(\psi)$ results in the probability $P_{\lambda}$ that it contains three null lines separated by $60^{\circ}$ on the plane where the $\boldsymbol{r}$ vector lies. These null lines correspond to the degeneracies $\lambda_{1}=\lambda_{2}, \lambda_{2}=\lambda_{3}$, and $\lambda_{3}=\lambda_{1}$. This feature causes the random angle $\psi$ to distribute nonuniformly, but with threefold symmetry.

On changing the variables from $\left(\lambda_{1}, \lambda_{2}, \lambda_{3}\right)$ to $(\delta, r, \psi)$, the $\delta, \tilde{I}$, and $r$ integrals can all be evaluated analytically, and equation (4) now becomes

$$
P(j)=w^{-1} j^{3} \int \frac{d^{3} \Omega}{Y^{4}} K_{1}\left(\sqrt{\frac{5}{2}} \frac{j}{b Y}\right) J(\psi),
$$

where $d^{3} \Omega \equiv \sin \theta d \theta d \phi d \psi, K_{1}$ is the first-order modified Bessel function, $b \equiv\left(\sigma_{\delta} \sigma_{\tilde{I}}\right)^{1 / 2}, \quad w$ is the normalization factor, and $Y$ is given in equation (3). As $\log (j / b)=\log (j)-\log (b)$, the shape of the $\log (j)$ distribution is not affected by the value of the unknown $b$, although the peak position is. The angular integration of equation (6) can only be evaluated numerically, and the solid line of Figure 3 presents the resulting $j P(j)$. It shows that although the main body of $P(j)$ resembles a lognormal distribution (dashed line), the distribution is not exactly lognormal. One can check from equation (6) by asymptotic expansion that $P(j)$ drops off exponentially at the high angular momentum limit (e.g., Maller et al. 2002) and behaves as a power law $\left(\sim j^{2}\right)$ in the lower angular momentum limit. The best-fit lognormal to our distribution has a $\log$ width $\sigma_{\log j}=0.63$ (dashed line), which is in fair agreement with the log width $\sigma_{\log j}=0.5$ obtained from $N$-body simulations reported by Bullock et al. (2001). (Their definition of $\lambda^{\prime}$ is equivalent to our $j$ modulo a constant factor.)

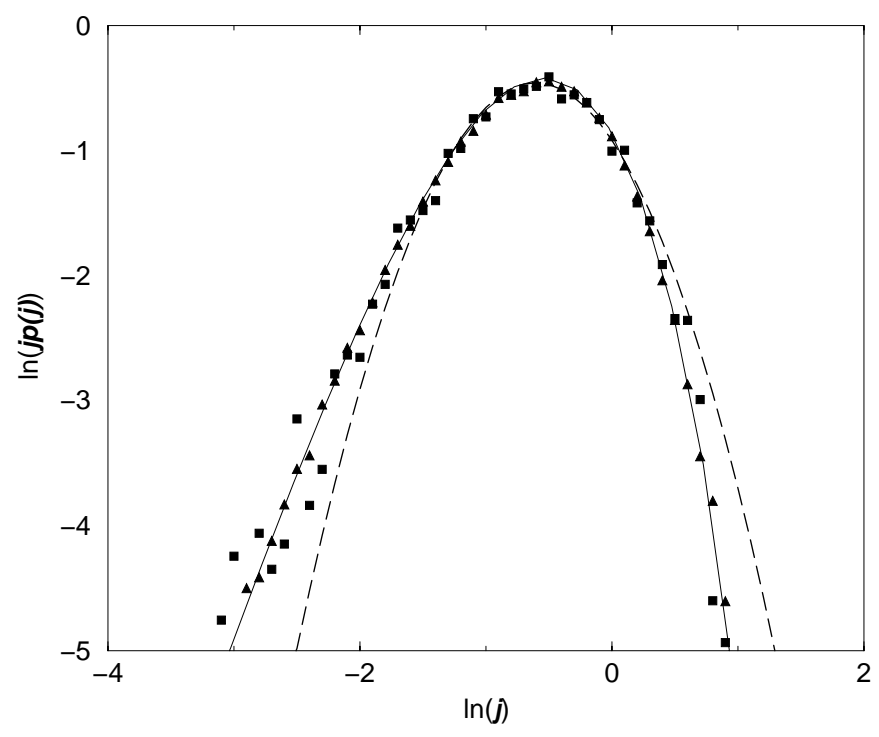

FIG. 3.-Distribution of the logarithmic specific angular momentum of dark halos, $j P(j)$. The solid curve shows our analytic result given by eq. (6); the squares show the Monte Carlo result for the nonspherical collapse model, and the triangles show the Monte Carlo result for the PressSchechter model. The dashed line shows the best lognormal fit to $j P(j)$.

For comparison, we have also simulated nonspherical halo collapse using the random walk algorithm with the $y_{j}$ variables. When the nonspherical collapse condition given in $\S 3.1$ is satisfied, we reconstruct the local shear tensor and diagonalize it to obtain the three unordered eigenvalues $\lambda_{i}$ (Chiueh \& Lee 2001). The angular momentum of the dark halo is then computed according to equation (1). Figure 3 also plots the resulting $j P(j)$ (squares). To ensure the accuracy of our method, we have also repeated the same random walk calculation for the Press-Schechter collapse condition and computed its $j P(j)$ (triangles), which should coincide with the solid line obtained analytically. As one can see, all three results agree with each other, revealing that the angular momentum distribution is insensitive to the detailed dynamics of halo formation.

We would like to stress that the unique shape and $\log (j)$ width of the angular momentum distribution are the direct outcome of our model. They involve no fitting parameter.

\section{DISCUSSION AND CONCLUSIONS}

To explain the observed characteristic shape for the angular momentum distribution of dark halos in $N$-body simulations, we have adopted the standard tidal-torque theory to study the specific angular momentum of a protohalo, $j$, which can be succinctly represented by a random shear $\mathrm{R}_{i j}$ acting on a random vector $\tilde{I}_{i}$. The diagonalized $\mathrm{R}_{i j}$, whose three components are the mutual differences of the three eigenvalues of the local shear tensor, quantifies the nonsphericality of the local gravitational potential, and the vector $\tilde{\boldsymbol{I}}$ characterizes the misalignment between the matter distribution and the local shear. With this picture, we have derived a new expression for the halo specific angular momentum, which separates the variables into three parts, $r, \tilde{I}$, and $Y(\psi, \theta, \phi)$. With $P_{r}(r \mid$ halo $)$ being shown to be approximately a weighted Gaussian and assuming the offdiagonal components of the inertia tensor in the shear principal-axis frame to also be Gaussian variables, we have 
derived the angular momentum distribution, i.e., $P(j)$, to be close to lognormal, with a mass-independent width in fair agreement with that determined from simulations. This "quasi"-lognormal distribution is simply a consequence of the nonlinear coupling of five random variables, $r, \tilde{I}, \theta, \phi$, and $\psi$.

Although the distribution of $\log (j)$ has a mass-independent shape, the mean of $\log (j)$ is predicted to depend on $b$ $\left[\equiv \sigma_{\tilde{I}} \sigma_{\delta}(M)\right]$. As our framework does not permit the mass dependence of $\sigma_{\tilde{I}}$ to be determined, the present work can only account for the ubiquitous shape, but not the peak location, of the angular momentum distribution. Nonetheless, simulations have empirically found that even the mean value of $\log (j)$ is also mass independent (Barnes \& Efstathiou 1987; Heavens \& Peacock 1988; Lemson \& Kauffmann 1999; Bullock et al. 2001; Chen \& Jing 2002; Vitvitska et al. 2002). Incorporating this finding, we thus predict that

$$
\sigma_{\widetilde{I}}(M) \propto \sigma_{\delta}^{-1}(M)
$$

That is, the magnitude of the mass-independent rescaled inertia tensor $\tilde{I}$ has a wider distribution for larger halos than for smaller halos at the same epoch, since $\sigma_{\delta}$ is greater for smaller halos than for larger halos.
To make sense of this prediction, we conjecture that the following picture may be what happens. At a given epoch, a large halo has undergone vigorous growth with a high probability to have undergone merging events in the past. That is, chaotic dynamics make it possible to capture distant halos. The infall of merged subhalos renders the halo Lagrangian boundary irregular, where the halo inertia tensor and shear tensor can become significantly decorrelated. By contrast, a small halo grows mildly and tends to be in a quiescent low background density environment, thus keeping itself well correlated with the surrounding matter throughout the evolution. This picture yields the qualitative trend implied by equation (7). In any case, equation (7) as well as the Gaussianity assumption for $\tilde{I}$ are the tests of the present model, and they can both be scrutinized by cosmological $N$-body simulations.

This work has been supported by the Taida-ASIAA CosPA Project. T. C. acknowledges partial support from the National Science Council of Taiwan under grant NSC90-2112-M-002-026.
Barnes, J., \& Efstathiou, G. 1987, ApJ, 319, 575

Bullock, J. S., Dekel, A., Kolatt, T. S., Kravtsov, A. V., Klypin, A. A.

Porciani, C., \& Primack, J. R. 2001, ApJ, 555, 240

Catelan, P. \& Theuns, T. 1996, MNRAS, 282, 436

Chen, D. N., \& Jing, Y. P. 2002, MNRAS, 336, 55

Chiueh, T., \& Lee, J. 2001, ApJ, 555, 83

Chiueh, T., \& Tseng, Y. H. 2000, ApJ, 544, 204

Doroshkevich, A. G. 1970, Astrofizika, 6, 581

Frenk, C. S., White, S. D. M., Davis, M., \& Efsathiou, G. 1988, ApJ, 327, 507

Heavens, A. F \& Peacock, J. A. 1988, MNRAS, 232, 339

Lee, J., \& Pen, U. 2000, ApJ, 532, L5

Lemson, G., \& Kauffmann, G. 1999, MNRAS, 302, 111

\section{REFERENCES}

Lin, L., Chiueh, T., \& Lee, J. 2002, ApJ, 574, 527

Maller, A. H., Dekel, A., \& Somerville, R. S. 2002, MNRAS, 329, 423

Porciani, C., Dekel, A., \& Hoffman, Y. 2002a, MNRAS, 332, 325 2002b, MNRAS, 332, 339

Press, W. H., \& Schechter, P. 1974, ApJ, 187, 425

Sheth, R. K., Mo, H. J., \& Tormen, G. 2001, MNRAS, 323, 1

Sheth, R. K., \& Tormen, G. 1999, MNRAS, 308, 119 2002, MNRAS, 329, 61

Sugerman, B., Summers, F. J., \& Kamionkowski, M. 2000, MNRAS, 311, 762

Vitvitska, M., Klypin, A. A., Kravtsov, A. V., Wechsler, R. H., Primack, J. R., \& Bullock, J. S. 2002, ApJ, 581, 799

White, S. D. M. 1984, ApJ, 286, 38 